\title{
Thoracic Surgery during Coronavirus Disease 2019 (COVID-19): The Experience of a Level 1 Trauma Center
}

\author{
Jeremy Smelt ${ }^{1}$ Gowthanan Santhirakumaran ${ }^{1}$ Paul Vaughan ${ }^{1}$ Ian Hunt ${ }^{1}$ Carol Tan ${ }^{1}$ \\ ${ }^{1}$ Department of Thoracic Surgery, St. George's University Hospitals \\ NHS Foundation Trust, London, United Kingdom \\ Thorac Cardiovasc Surg 2021;69:252-258. \\ Address for correspondence Jeremy Smelt, FRCS(CTh), PhD, \\ Department of Thoracic Surgery, St George's University Hospitals \\ NHS Foundation Trust, Blackshaw Road Tooting, London SW17 0QT, \\ United Kingdom (e-mail: jeremysmelt@doctors.org.uk).
}

\begin{abstract}
Background Severe acute respiratory syndrome coronavirus 2 (SARS-CoV-2), a novel coronavirus primarily affecting the respiratory system, was initially diagnosed in Wuhan, China, in late 2019. Identified as coronavirus disease 2019 (COVID-19) by the World Health Organization, the virus rapidly became a global pandemic. The effects on health care worldwide were unprecedented as countries adapted services to treat masses of critically ill patients.

The aim of this study is to analyze the effect that the COVID-19 pandemic had on thoracic surgery at a major trauma center during peak prevalence.

Methods Prospective unit data were collected for all patients who underwent thoracic surgery during March 2020 until May 2020 inclusive. Retrospective data were collected from an earlier comparable time period as a comparison.

Results In the aforementioned time frame, 117 thoracic surgical operations were performed under the care of four thoracic surgeons. Six operations were performed on three patients who were being treated for SARS-CoV-2. One operation was performed on a patient who had recovered from SARS-CoV-2. There were no deaths due to SARSCoV-2 in any patient undergoing thoracic surgery.

Keywords

- COVID-19

- coronavirus

- pandemic

- thoracic surgery

- lung cancer

Conclusion This study demonstrates that during the first surge of SARS-CoV-2, it was possible to adapt a thoracic oncology and trauma service without increase in mortality due to COVID-19. This was only possible due to a significant reduction in trauma referrals, cessation of benign and elective work, and the more stringent reprioritization of cancer surgery. This information is vital to learn from our experience and prepare for the predicted second surge and any similar future pandemics we might face.
\end{abstract}

\section{Introduction}

An outbreak of similar but unusual cases of pneumonia was seen in Wuhan, Hubei Province, China, toward the end of $2019 .^{1}$ These patients, it emerged, were the beginning of what would be declared a global pandemic of a novel coronavirus, severe acute respiratory syndrome coronavirus 2 (SARS-CoV-2), by March 2020. ${ }^{2}$ The World Health Organi-

received

July 20, 2020

accepted after revision

August 27, 2020

published online

November 20, 2020

zation (WHO) named the disease caused by the enveloped RNA $\beta$ coronavirus, COVID-19. ${ }^{3}$ By the end of May 2020, more than 6 million people worldwide had been diagnosed with the virus, and 368,993 people had died as a result of COVID$19 .{ }^{4}$ In the United Kingdom, 153,538 people had been diagnosed with the virus, and 39,010 had died from the disease. ${ }^{5}$

SARS-CoV-2 primarily affects the lungs, causing widespread initial changes and respiratory failure. ${ }^{1,6,7}$ Patients

(c) 2020. Thieme. All rights reserved.

Georg Thieme Verlag KG,

Rüdigerstraße 14,

70469 Stuttgart, Germany
DOI https://doi.org/ 10.1055/s-0040-1718938. ISSN 0171-6425. 
present with common symptoms such as cough and fever; however, like other coronaviruses, SARS-CoV-2 has a spectrum of severity, with some patients having minimal or no symptoms and others becoming critically unwell. Certain comorbidities and demographics, such as increased age, male sex, and diabetes, were found to be related to increased severity of the infection and poor prognosis. ${ }^{8}$ A symptomfree incubation period of around 1 week followed by variable types and degree of symptoms as well as high risk of aerosol spread makes the virus highly contagious. ${ }^{9-11}$ A "cytokine storm" after around a week of symptoms is thought to be the causation of lung parenchymal infiltration and resulting respiratory deterioration and possible failure. ${ }^{12}$ Initially, diagnosis was based on clinical assessment, especially temperature measurements, and symptom history along with contact tracing information. This was supported by radiological evidence of pulmonary changes in keeping with COVID-19. Eventually, a postnasal and oropharyngeal swab detected SARS-CoV-2 by reverse transcription-polymerase chain reaction (RT-PCR). ${ }^{13,14}$

Health care systems worldwide were therefore obliged to try and rapidly adapt their services to absorb this new burden of disease. The National Health Service (NHS) was required almost overnight to expand its critical care capacity and workforce by redistributing resources. Other specialties had to rapidly adapt and prioritize their services with little guidance. ${ }^{15}$ There was emerging evidence from countries that already had a high disease burden, such as China and Italy, suggesting that patients undergoing thoracic surgery in the context of COVID-19 had a poor prognosis. ${ }^{16}$ Furthermore, patients with thoracic malignancies who developed COVID-19 had higher than baseline risk of complication and death. ${ }^{17}$ There was also evidence to suggest that nosocomial infections were a major issue and responsible for significant viral spread. ${ }^{18}$ All of these factors meant that thoracic surgery, especially in cancer patients, was a high-risk affair. This also led to ethical dilemmas affecting patient selection. ${ }^{19}$

To prevent the spread of disease, countries imposed varying sanctions on activities. The United Kingdom government imposed social distancing laws in March followed by an enforced "Lockdown" a week later. This "lockdown" prevented any nonessential activities, travel, and work from being performed. $^{20}$

At our institution, 992 patients who tested positive for SARS-CoV-2 were treated as inpatients. Of these, 295 (29.7\%) died. The South London Organization Delivery Network, to which our intensive care belongs, had the highest number of admissions to critical care in the United Kingdom. ${ }^{8}$ There were 60 intensive care beds at our hospital prior to the pandemic, but within 2 weeks of admitting the first patient with SARS-CoV-2, the number of beds doubled, with plans in place to increase to 200 if required. The majority of patients with COVID-19 were admitted and treated within the 3month window of March, April, and May 2020.

The aim of this study is to analyze the effect that the COVID-19 pandemic had on thoracic surgery at a major trauma center during the peak prevalence of this disease. There is widely expected to be a second surge of this virus. ${ }^{21}$
This information is vital to learn from our experience and prepare for the predicted second surge and any similar future pandemics we might face.

\section{Materials and Methods}

Prospective unit data collected for all patients who underwent thoracic surgery during March 2020 until May 2020 inclusive were analyzed. This data included demographic information including operative details and outcome measures such as length of stay, complications, and readmission. Follow-up of these patients was a minimum of 4 weeks.

Retrospective unit data were analyzed using the national cardiothoracic society audit returns for a 3-month period in the preceding year as an estimation of typical unit workload.

\section{Screening Procedures for COVID-19}

Elective Patients Up to and Including March 29, 2020

Patients undergoing surgery up to and including March 29, 2020, were investigated for COVID-19 on admission using a chest radiograph examining for bilateral infiltrates, a temperature recording of more than $37.6^{\circ} \mathrm{C}$, and positive symptom interrogation. New continuous cough and fevers were symptoms seen as pathognomonic symptoms for COVID-19. If the patient did not screen positive for these examinations, then the patient was deemed COVID-19 negative. These patients were designated as "green" and were managed in an open "green" ward and operated in the "green" operating rooms.

Patients undergoing surgery up to and including March 29, 2020, who were screened positive would have undergone a nasopharyngeal and oropharyngeal swab to detect the SARSCoV-2 virus through RT-PCR analysis. The patient would not have been admitted and surgery postponed while pending swab result.

Emergency Patients Up to and Including March 29, 2020 Patients who required emergency surgery were treated as COVID-19 unknown status and were managed in an "amber" ward. Due to operating rooms capacity, the patients would be operated on in "red" operating rooms (operating rooms that also treated proven COVID-19 positive patients).

Subsequent COVID-19 screening would take place including temperature measurement, chest radiograph, and symptom interrogation. All patients admitted to this route would also have a nasopharyngeal and oropharyngeal swab test to detect the SARS-CoV-2 virus through RT-PCR.

If the screening tests were negative, then the patient would have moved onto the "green" areas. If found to have a positive swab or high suspicion based on the other screening measures, then the patient would have moved to a "red" or COVID-19 positive ward and treated in "red" operating rooms.

\section{Elective Patients Up to and Including May 3, 2020}

These patients underwent the usual clinical and radiological screening tests with the addition of a nasopharyngeal and oropharyngeal swab to detect the SARS-CoV-2 virus via RTPCR analysis. Patients underwent this swab 3 days prior to 
their operation using a "drive-through" service to ensure that the result was available prior to surgery.

If these tests were negative, then the patient was designated as "green" and followed the "green" pathway. If it was positive, then the patient would not have been admitted and the surgery deferred.

Emergency Patients Up to and Including May 3, 2020 The only change to the protocol here was the introduction of "amber" operating rooms. Patients who had COVID-19 unknown status underwent surgery in "amber" operating rooms.

\section{Elective Patients from May 4, 2020}

These patients underwent the usual clinical and radiological screening tests with the addition of a nasopharyngeal and oropharyngeal swab to detect the SARS-CoV-2 virus via RTPCR analysis. However, these patients were also asked to shield for 2 weeks prior to admission. Shielding was defined as not leaving the patient's dwelling and socially distancing at $2 \mathrm{~m}$ from all occupants. These patients (if shielded and negative on screening) were placed in a "supergreen" ward. If shielding was not performed but screening was negative, then the patient would be admitted to a "green" ward instead.

Emergency Patients from May 4, 2020

There was no change to this protocol after this date.

\section{Operating Room Protocols}

All patients were operated on with all staff wearing full personal protective equipment (PPE), which included operating room hat, FFP3 mask, face visor, double sterile gloves, and gown. After each procedure in a "red" or "amber" operating room, a "deep clean" was performed prior to further surgery. After both intubation and extubation, which were performed with minimal staff in the operating room (anesthetist and operating department assistant), staff waited 20 minutes before entering, leaving, or moving the patient from the operating room.

\section{Facemask Use}

Patients were only required to use facemasks when being transported around the hospital. Staff were obliged to wear facemasks when in "green" or "supergreen" wards, and FFP3 masks and equipment were mandatory on "amber" or "red" wards.

\section{Visiting}

During these three months, there was no visiting allowed in the hospital.

\section{Communication}

Daily "Coronavirus Bulletins" were sent to staff within the hospital with updated guidelines on PPE and COVID-19 protocols for the majority of the 3 months. Weekly meetings with clinical leads from each department within the hospital and senior management staff called when new advice, protocols, and guidelines were discussed and disseminated.
Table 1 Summary of operations performed between March and May 2020 inclusive

\begin{tabular}{|l|l|}
\hline Operation & $N(\%)$ \\
\hline VATS lobectomy & $17(14.5 \%)$ \\
\hline VATS for empyema & $16(13.7 \%)$ \\
\hline VATS for pneumothorax & $11(9.4 \%)$ \\
\hline VATS wedge resections & $10(8.5 \%)$ \\
\hline VATS pleural biopsies & $10(8.5 \%)$ \\
\hline Mediastinoscopies & $10(8.5 \%)$ \\
\hline Open lobectomy & $8(6.8 \%)$ \\
\hline Washout for hemothorax & $8(6.8 \%)$ \\
\hline Rigid bronchoscopies & $4(3.4 \%)$ \\
\hline Open pneumonectomy & $1(8.5 \%)$ \\
\hline VATS bilobectomy & $1(8.5 \%)$ \\
\hline $\begin{array}{l}\text { Anterolateral thoracotomy for } \\
\text { traumatic tamponade }\end{array}$ & $1(8.5 \%)$ \\
\hline Rib fixation & $1(0.9 \%)$ \\
\hline Other & $19(16.2 \%)$ \\
\hline Total & $117(100 \%)$ \\
\hline
\end{tabular}

Abbreviation: VATS, video-assisted thoracoscopic surgery.

\section{Results}

A total of 117 thoracic surgical operations were performed in this time frame under the care of four thoracic surgeons. These were made up of 80 elective, 33 urgent, and 4 emergency cases (-Table $\mathbf{1}$ ).

\section{Lung Cancer and Other Lung Resections}

Twenty-seven elective anatomical lung resections for primary lung cancer were performed (23\% of all procedures), of these, $12(44.6 \%)$ patients were males and 15 (55.6\%) females, with a median age of 71 years (range: $36-79$ years). One (3.7\%) patient underwent neoadjuvant chemotherapy prior to lung resection. Also, 1 (3.7\%) pneumonectomy, 1 bilobectomy (3.7\%) and 25 (92.6\%) lobectomies were performed in this group. Three of the lobectomies had frozen sections to confirm the diagnosis intraoperatively, and there were no negative frozen sections. Eighteen operations were performed using video-assisted thoracoscopic surgery (VATS), and nine were performed through thoracotomy. Only two VATS procedures required conversion to thoracotomy due to difficulty to progress. Sixteen patients had stage 1 disease and eight had stage 2 disease. One patient had stage 3a disease with pT4 pN0, and two patients had stage $3 \mathrm{~b}$ disease with pT3 pN2. Both patients who had N2 disease were upstaged by surgical pathology. Three patients had a prolonged air leak (more than 7 days), one patient had atrial fibrillation, one patient had a chyle leak requiring prolonged drainage only, and one patient required antibiotics for a postoperative chest infection. None of the patients required an intensive care unit (ICU) stay, and one patient was readmitted for 5 days with surgical emphysema requiring a chest drain. 
During the time frame, 10 wedge resections (nonanatomical lung resection) were performed, which was $8.5 \%$ of all procedures: 6 were performed for curative primary lung resections, 2 were diagnostic (rheumatoid nodule and inflammatory nodule), and 2 were performed for metastatic oncological disease.

\section{Trauma and Other Emergency Operations}

Only four emergency thoracic operations were performed during this period. Two (50\%) patients required VATS for spontaneous hemothorax (both thought to be from a ruptured adhesion), one (25\%) patient required VATS for empyema causing a mediastinal shift, and one (25\%) patient required a left anterior thoracotomy for pericardial tamponade from a stab wound. One patient underwent rib fixation.

\section{SARS-COV-2}

Three patients were operated on an urgent basis for hemopneumothoraces resulting from the combined effect of COVID-19, coagulopathy, and prolonged positive-pressure ventilation. All three of these patients required a return to the operating room 24 to 72 hours later for further washout and arrest of bleeding. These three patients were all subsequently discharged from the hospital after prolonged intensive care and inhospital stays.

One patient underwent a mediastinoscopy having previously tested positive for COVID-19 and had a brief hospital stay for noninvasive ventilation 5 weeks prior. A repeat RT-PCR swab 3 weeks after an initial positive swab returned a positive result. Surgery was therefore postponed for 2 weeks until a total of 5 weeks after the initial swab test. There were no deaths due to COVID-19 in any patient undergoing thoracic surgery. No patients who underwent thoracic surgery subsequently developed COVID-19 within 6 weeks of surgery.

\section{Further Surgery}

Of the 117 operations performed during this period, 10 (8.5\%) were cervical mediastinoscopies, which is not in keeping with usual practice. This increase in activity was due to endobronchial ultrasound not being readily available due to the concern that it is an aerosol-generating procedure.

The majority of the remaining operations (37 [31.6\%]) were for empyema and pneumothorax that had failed medical management, including VATS pleural biopsies for the diagnosis and management of effusions.

As a comparison of typical unit workload, data from the Society for Cardiothoracic Surgery National Audit Returns for our unit suggested that during a 3-month period, our unit would perform 212 operations, of which 44 (20.8\%) would be anatomical elective lung resections, 12 (5.6\%) wedge resections for lung cancer, 10 (4.7\%) mediastinoscopies, and 7 (3.3\%) rib fixations (-Fig. $\mathbf{1}$ ).

\section{Discussion}

\section{Operative Numbers}

Compared with other specialties within our hospital, which was reflected NHS wide, cancer services and especially

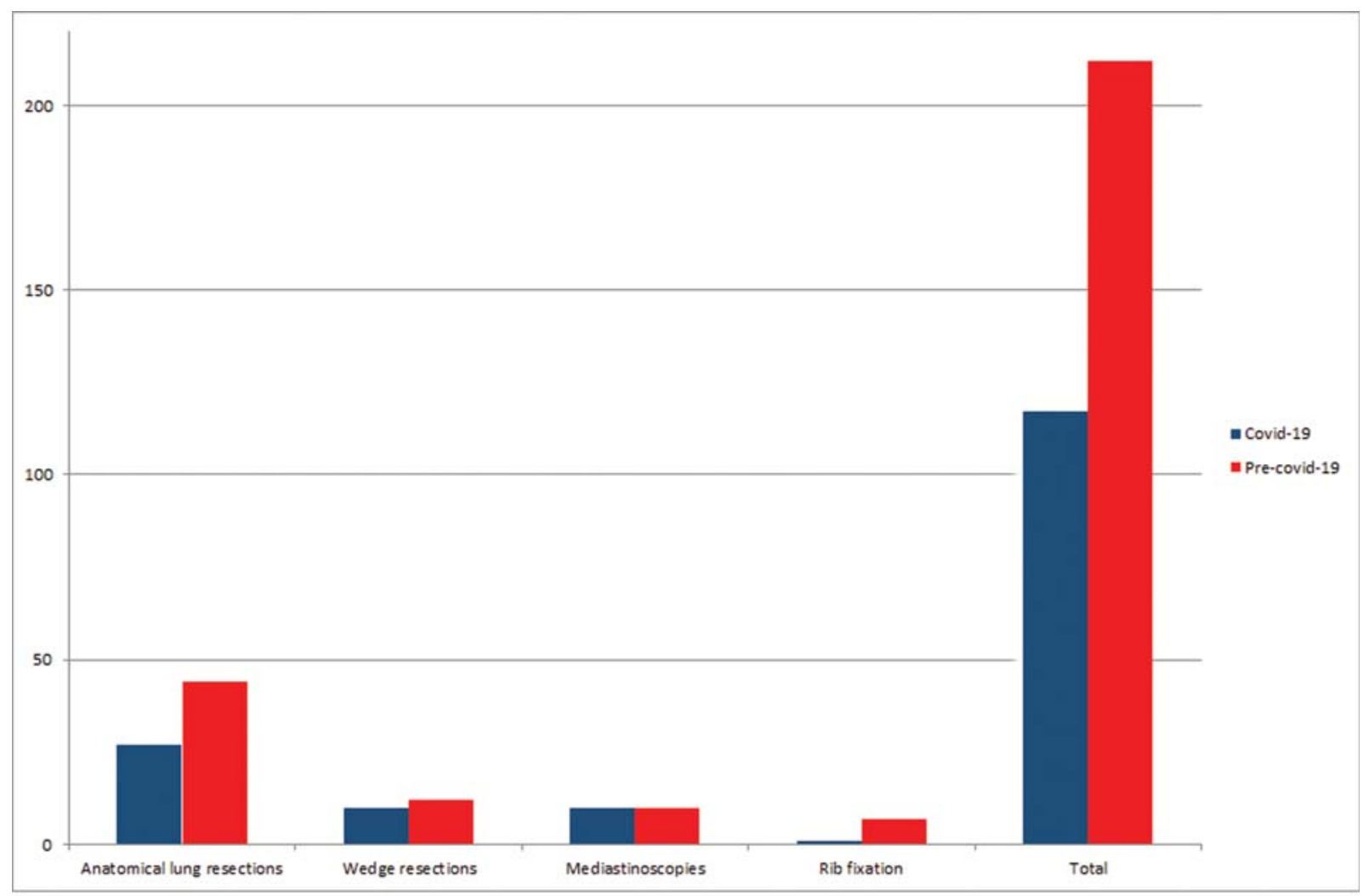

Fig. 1 Comparison between workloads during the COVID-19 era (March to May 2020) and representative pre-COVID-19 era. COVID, coronavirus disease 2019. 
aggressive tumor types such as lung cancers were given high priority to continue their activity. This is reflected by the workload described previously. This workload was actively protected within the hospital by ensuring access to operating room space and perioperative beds. Furthermore, multiple protocols were instituted to provide patient pathways that limited the risk of patient exposure to SARS-CoV-2 in the hospital as well as to reduce the risk of patients entering the hospital with the infection. These protocols changed throughout the time period analyzed and adapted to suit guidelines, emerging evidence, and government advice.

However, there was still a reduction in activity during this time. Reasonably, there was great concern as to the outcomes of patients undergoing surgery. ${ }^{22}$ There was a paucity of evidence on the subject due to its novel nature, and the disease primarily affected the lungs. Therefore, the approach was to limit surgery to immediate necessity only.

With some operating room spaces being converted into temporary ICUs, the number of operating room sessions available for thoracic surgery was reduced from 5 days to 2 days a week over the 3-month period. In addition, there was also prolonged turnaround time between patients in the operating room due to new protocols, and fewer cases than previously were therefore scheduled in each operating session. These two organizational issues also contributed to the reduction in thoracic surgical activity.

Initially, during March 2020, when the first patients were admitted to the hospital with evidence of the SARS-CoV-2 infection, clinicians were asked to categorize any patients for surgery through both the NHS and National Cancer Guidelines. ${ }^{23,24}$ Surgery was therefore offered to patients who did not have a good alternative and who required surgery within 3 months to prolong their life. Almost no benign elective procedures were therefore performed in this period. The prioritization for surgery and guidelines were distributed to peripheral referring centers, which continued to have weekly multidisciplinary team (MDT) meetings with thoracic surgical consultants in attendance. Due to the referral and diagnostic pathway length, there were around 6 weeks before a drop in surgical referrals was noticed in the MDTs. This drop was thought to be due to stricter 2-week wait referrals, and symptomatic patients avoiding health care settings. ${ }^{25}$

Video linking into MDTs and video or telephone consultations was implemented to reduce footfall to peripheral hospitals for both surgeons and patients to decrease the spread of the virus. Initially, there was a reluctance to adopt this by the surgeons as it was thought to increase the difficulty of assessing surgical risk and relying on meeting the patient in person on admission; however, there were no patients who were deemed not fit for surgery at subsequent attendance of preassessment clinic or on admission and the practice continued throughout and beyond the 3 months.

\section{Screening and Perioperative Protocols}

Early in the screening protocol, without the use of a nasopharyngeal and oropharyngeal swab to detect the SARSCoV-2 virus through RT-PCR analysis, there was theoretically the highest chance that a patient could have had elective surgery while suffering from the SARS-CoV-2 infection with potentially devastating consequences. However, at this time, there were thought to be a low, but increasing prevalence within the community, based on numbers of SARS-CoV-2 infections within the hospital and community swab testing, but increasing prevalence within the community, based on numbers of SARS-CoV-2 infections within the hospital and community swab testing. The reason for not introducing the swab earlier was simply because it was not available for this use at the time. The patients who underwent operations at this time were reprioritized in light of the COVID-19 risk and were deemed to not have a suitable alternative treatment and not to be able to have this treatment postponed.

This radical and evolving change in practice over these few months required staff to adapt rapidly. The decrease in patient episodes was offset by the redeployment of staff into other roles from the specialty of thoracic surgery as well as staff illness with SARS-CoV-2 infection (contact tracing did not demonstrate an obvious source). During these 3 months, at any one time, there were three consultants providing 24 hours cover to the service and three junior staff providing daytime cover. The logistic difficulties of planning these operations in this era made the nonclinical workload increase substantially, whereas the nonclinical staff members were encouraged to work remotely from home. Despite these challenges, surgical cancellation and complication rates remained low in keeping with prepandemic practice.

\section{Trauma Service}

The dramatic decrease in trauma activity is thought to be due to the U.K.-wide "lockdown" resulting in the reduction in activities that create trauma (driving, manual labor, highrisk recreational sports, and public gatherings) ${ }^{26}$ Certainly, this would account almost entirely for the decrease in the number of emergency procedures required for hemostasis following trauma; however, this may not explain the decrease in the number of urgent procedures for trauma such as rib fixation. It is likely that during this period, a more conservative approach was taken in the management of blunt chest trauma, which is more prevalent. The patients who underwent rib fixation had displaced ribs, a hemothorax despite a chest drain, and poor pain control despite maximal analgesia including a serratus anterior block.

\section{Procedural Aberrations}

The number of wedge resections for lung cancer was predicted by the authors to increase during the SARS-CoV-2 outbreak. Similar to operating on higher risk or elderly population, an attempt to reduce the perceived operative risk and potential complications and therefore reduction in hospital stay, the surgeon may elect to perform a wedge instead of an anatomical lung resection. ${ }^{27}$ In addition to surgeon preference, there were also two patients who elected to have wedge resections rather than anatomical lung resection to reduce perioperative risk. However, lesions that were amenable to a wedge resection, early stage peripheral tumors less than $2 \mathrm{~cm}$, would likely have been referred for stereotactic ablative radiation therapy (SABR) as an alternative treatment modality. Frozen section analysis 
was also thought to increase in prevalence due to the limitation in access to computed tomography (CT) guided biopsy; however, this was not seen in this patient cohort, although numbers were limited. An explanation for this was a tendency to follow up indeterminate early stage lesions or refer for SABR on the basis of PET (positron emission tomography) avidity and interval growth, in line with the contemporary guidance.

\section{Thoracic Surgical Complications of SARS-COV-2}

Three specific complications were noted to develop as a result of patients being ventilated for respiratory failure secondary to SARS-CoV-2 infection and had a high incidence in this group of patients. These were pneumothorax, pneumomediastinum, and spontaneous hemothorax.

Pneumothorax was seen in ventilated patients requiring high pressures and was thought to be related to a combination of barotrauma and parenchymal destruction of the lung secondary to the disease itself. $^{28}$ These cases were managed conservatively with the insertion of an ipsilateral chest drain.

Pneumomediastinum was also seen in this cohort of intubated patients with SARS-CoV-2 disease. The predominant sign was sudden onset surgical emphysema and subsequent CTconfirmed surgical emphysema associated with free mediastinal air. This was rarely associated with pneumothorax. This was managed expectantly; however, if a pneumothorax was identified, then placement of chest drain was performed. The occurrence of pneumomediastinum was a poor prognostic sign, and the majority of these patients died.

Spontaneous hemothorax was thought to result from a combination of pneumothorax, secondary to SARS-CoV-2 lung disease and positive pressure, and coagulopathy associated with the virus. Histopathology from SARS-CoV-2 positive patients in Wuhan demonstrated a high burden of microscopic thromboembolic disease throughout the lung vasculature. $^{29}$ This progressed to high pulmonary artery pressure, right-sided heart strain, and demonstrable pulmonary emboli on contrast CT scanning in many patients in our institution. As a result of this, patients were treated with systemic heparin therapy. In addition, many patients required treatment with renal replacement therapy, which required heparin administration. Of the three patients who were stable enough for the operating room, cessation of bleeding was not possible surgically due to widespread hemorrhage from the pleural surface. Hemostasis was achieved by clearing out blood and clot, placing multiple drains, and placing these on suction to allow the lung to tamponade against the chest wall. All three patients required a return to the operating room to repeat the procedure after recollecting into the pleural cavity.

\section{Future Challenges}

There lies ahead a difficult balance. Firstly, the decrease, but not eradication, of SARS-CoV-2 prevalence in the community requires protocols to remain in place and a high level of caution in reducing the rapidly instituted measures employed in these few months to prevent nosocomial transmission. Secondly, flexibility and readiness have to be maintained to rapidly introduce stringent safety protocols in the event of the predicted second surge.

Research should be rapidly directed toward patient outcomes for those affected by the reduction in available oncological and trauma surgery in order to quantify harm caused as a result. This can help rationalize the changes we make during the pandemic.

It is known from previous evidence that SARS-CoV-2 virus infection in both lung cancer patients and patients undergoing thoracic surgery can have devastating effects. It is important, however, to continue to provide life-saving surgery in patients who have lung cancers not amenable to other less invasive treatments and who cannot wait until the pandemic resolves. Furthermore, emergency thoracic surgery cannot be deferred for trauma and life-threatening conditions.

This study demonstrates that it is possible to continue a thoracic oncological and trauma service during the peak of a COVID-19 pandemic with the understanding that strict adherence to both government and health care (local and national) guidelines and protocols are required to minimize the effect of COVID-19 perioperatively. The description and adaptation of the protocols described should be helpful to other centers that require the continuation of a thoracic surgical service during this and future pandemics. Furthermore, we have described common thoracic surgical complications of COVID-19 that surgeons must be aware of and be prepared to treat.

\section{Conclusions}

This study demonstrates that during the first surge of SARSCoV-2, it was possible to maintain a thoracic oncology and trauma service without increase in mortality due to the virus. However, this service required fluid protocol adaptations that were both time-consuming and difficult to implement. Furthermore, this was only possible due to a significant reduction in trauma referrals, cessation of benign and elective work, and the more stringent reprioritization of cancer surgery. Future research will be required to analyze the effect this change will have had on patients. This information is vital to learn from our experience and prepare for the predicted second surge and any similar future pandemics we might face.

\section{Funding}

There are no sources of funding for the work to declare.

Conflict of Interest

There are no conflicts of interest to declare.

\section{References}

1 Huang C, Wang Y, Li X, et al. Clinical features of patients infected with 2019 novel coronavirus in Wuhan, China. Lancet 2020;395 (10223):497-506

2 World Health Organization WHO Director-General's opening remarks at the media briefing on COVID-19 - 11 March 2020 https://www.who.int/dg/speeches/detail/who-director-general-sopening-remarks-at-the-media-briefing-on-covid-19-11-march2020. Accessed July 2, 2020. 
3 World Health Organization Novel Coronavirus (2019-nCoV): Situation Report - 22. https://www.who.int/docs/default-source/ coronaviruse/situation-reports/20200211-sitrep-22-ncov.pdf? sfvrsn=fb6d49b1_2. Accessed July 2, 2020

4 John Hopkins University of Medicine Coronavirus Resource Center. https://coronavirus.jhu.edu/. Accessed July 2, 2020

5 Government of UK Coronavirus (COVID-19) cases in the UK. https://coronavirus.data.gov.uk/. Accessed July 2, 2020

6 Guan WJ, Ni ZY, Hu YChina Medical Treatment Expert Group for Covid-19, et al. Clinical characteristics of coronavirus disease 2019 in China. N Engl J Med 2020;382(18):1708-1720

7 Grasselli G, Zangrillo A, Zanella ACOVID-19 Lombardy ICU Network, et al. Baseline characteristics and outcomes of 1591 patients infected with SARS-CoV-2 admitted to ICUs of the Lombardy region, Italy. JAMA 2020;323:1574-1581

8 Intensive Care National Audit \& Research Centre ICNARC report on COVID-19 in critical care: 26 June 2020. https://www.icnarc.org/ DataServices/Attachments/Download/0a0738a6-dcb7-ea119127-00505601089b. Accessed June 30, 2020

9 Yu P, Zhu J, Zhang Z, Han Y, Huang L. A familial cluster of infection associated with the 2019 novel coronavirus indicating potential person-to-person transmission during the incubation period. J Infect Dis 2020;221(11):1757-1761

10 Li Q Guan X, Wu P, et al. Early transmission dynamics in Wuhan, China, of novel coronavirus-infected pneumonia. N Engl J Med 2020;382(13):1199-1207

11 Pung R, Chiew CJ, Young BESingapore 2019 Novel Coronavirus Outbreak Research Team. , et al; . Investigation of three clusters of COVID-19 in Singapore: implications for surveillance and response measures. Lancet 2020;395(10229):1039-1046

12 Zhang $\mathrm{C}$, Wu Z, Li JW, Zhao $\mathrm{H}$, Wang GQ. Cytokine release syndrome in severe COVID-19: interleukin-6 receptor antagonist tocilizumab may be the key to reduce mortality. Int J Antimicrob Agents 2020;55(05):105954

13 World Health Organization Global surveillance for COVID-19 caused by human infection with COVID-19 virus. https://www. who.int/docs/default-source/coronaviruse/2020-03-20-surveillance.pdf?sfvrsn=e6be6ef1_2. Accessed July 2, 2020

14 World Health Organization Laboratory testing for coronavirus disease (COVID-19) in suspected human cases. https:// apps.who.int/iris/rest/bitstreams/1272454/retrieve. Accessed July 2, 2020

15 COVIDSurg Collaborative. Global guidance for surgical care during the COVID-19 pandemic. Br J Surg 2020 (e-pub ahead of print). Doi: $10.1002 /$ bjs.11646

16 Cai Y, Hao Z, Gao Y, et al. Coronavirus disease 2019 in the perioperative period of lung resection: a brief report from a
Single Thoracic Surgery Department in Wuhan, People's Republic of China. J Thorac Oncol 2020;15(06):1065-1072

17 Garassino MC, Whisenant JG, Huang LCTERAVOLT investigators, et al. COVID-19 in patients with thoracic malignancies (TERAVOLT): first results of an international, registry-based, cohort study. Lancet Oncol 2020;21(07):914-922

18 Yu J, Wen Ouyang, Chua MLK, Xie C. SARS-CoV-2 transmission in patients with cancer at a tertiary care hospital in Wuhan, China. JAMA Oncol 2020;6(07):1108-1110

19 Harkin DW. Ethics for surgeons during the COVID-19 pandemic, review article. Ann Med Surg (Lond) 2020;55:316-319

20 Government of UK UK Government Guidelines on Coronavirus. https://www.gov.uk/government/publications/. Accessed July 2, 2020

21 Kissler SM, Tedijanto C, Goldstein E, Grad YH, Lipsitch M. Projecting the transmission dynamics of SARS-CoV-2 through the postpandemic period. Science 2020;368(6493):860-868

22 COVIDSurg Collaborative. Mortality and pulmonary complications in patients undergoing surgery with perioperative SARSCoV-2 infection: an International Cohort Study. Lancet 2020;396 (10243):27-38

23 Dingemans AC, Soo RA, Jazieh AR, et al. Treatment guidance for patients with lung cancer during the coronavirus 2019 pandemic. J Thorac Oncol 2020;15(07):1119-1136

24 National Health Services. Clinical guide for the management of noncoronavirus patients requiring acute treatment: Cancer. https://www.england.nhs.uk/coronavirus/wp-content/uploads/ sites/52/2020/03/specialty-guide-acute-treatment-cancer-23march-2020.pdf. Accessed July 2, 2020

25 Cancer Research UK How coronavirus is impacting cancer services in the UK. https://scienceblog.cancerresearchuk.org/2020/04/21/ how-coronavirus-is-impacting-cancer-services-in-the-uk/. Accessed July 2, 2020

26 Stinner DJ, Lebrun C, Hsu JR, Jahangir AA, Mir HR. The orthopaedic trauma service and COVID-19: Practice considerations to optimize outcomes and limit exposure. J Orthop Trauma 2020;34(07): 333-340

27 Smelt J, Lovejoy C, Thakker R, Hunt I, Martin F, Tan C. Elective lung resections in the elderly: where do we draw the line? Thorac Cardiovasc Surg. 2020(e-pub ahead of print). Doi: 10.1055/s-00393402725

28 Vega J, Gordo M, Tascón A, Vélez S. Pneumomediastinum and spontaneous pneumothorax as an extrapulmonary complication of COVID-19 disease. Emerg Radiol 2020(e-pub ahead of print). Doi: 10.1007/s10140-020-01806-0

29 Iba T, Levy J, Levi M, Connors J, Thachil J. Coagulopathy of coronavirus disease 2019. Crit Care Med 2020 (e-pub ahead of print). Doi: 10.1097/CCM.0000000000004458 